\title{
Three-dimensional imaging of steeply dipping structure near the San Andreas fault, Parkfield, California
}

\author{
John N. Louie*, Robert W. Clayton*, and Ronan J. LeBrasł
}

\begin{abstract}
Shot gathers from the Parkfield, California, deep crustal seismic reflection line, recorded in 1977 by COCORP, reveal coherent events having horizontal to reverse moveouts. These events were migrated using a multioffset three-dimensional Kirchhoff summation method. This method is a ray-equation back projection inversion of the acoustic wave field, which is valid under the Born, WKBJ, and far-field assumptions. Migration of full-wave acoustic synthetics, having the same limitations in geometric coverage as the COCORP survey, demonstrates the utility of the imaging process. The images obtained from back projection of the survey data suggest that the Gold Hill fault carries ultramafic rocks from the surface to $3 \mathrm{~km}$ depth at a dip greater than 45 degrees, where it joins the San Andreas fault, which may cut through more homogeneous materials at shallow depths. To the southwest, a $2 \mathrm{~km}$ Tertiary sedimentary section appears to terminate against a nearvertical fault. The zone between this fault and the San Andreas may be floored at $3 \mathrm{~km}$ by flat-lying ultramafics. Lateral velocity inhomogeneities are not accounted for in the migration but, in this case, do not seriously hinder the reconstruction of reflectors.
\end{abstract}

\section{INTRODUCTION}

The validity of the conventional process of stacking seismic reflection data as an imaging process depends on a number of assumptions about the character of the subsurface. Principally, the velocity of the medium must vary only slowly in the lateral direction. Where this constraint does not apply, a stacked section may not be interpretable. Some other method must be used to examine the data in the multioffset form of the physical seismic experiment.

This paper presents an example of a seismic reflection data set that cannot be fully interpreted using the stacking process and its underlying assumptions. It was recorded across a rather spectacular lateral heterogeneity, the San Andreas fault zone. It is seen below how the previous interpretations of this survey could not image the strongest reflections in the data set. To image these events, a method with less restrictive underlying assumptions is then described. Its effectiveness is demonstrated through imaging of synthetic data. Finally, the imaging of the field data set provokes conclusions on the relationship of the geologic and physical settings of both the area near the San Andreas fault and seismic reflection targets in general.

In 1977, COCORP recorded $27 \mathrm{~km}$ of deep crustal reflection data on a route crossing the San Andreas fault in Monterey County, California, near the town of Parkfield. This section of the fault has long been of interest to seismologists because of the regular occurrence of moderate earthquakes on it. Evaluations of velocities and other seismic characteristics of the region have been included in several studies of seismic activity, such as those by Eaton et al. (1970) and Liu (1983). Analysis of reflection profiles just to the north, along the San Andreas in San Benito County, by Feng and McEvilly (1983) shows that the fault zone is marked by "extreme lateral heterogeneity." This is principally expressed as relatively low velocities within a zone surrounding the fault a few kilometers wide.

Figure 1 is a map of the Parkfield area showing the route of the survey and major fault traces in the vicinity, as mapped by Hanna et al. (1972). The data from the COCORP survey were originally processed and interpreted by Long (1981). He made interpretations of the history of the crustal blocks juxtaposed by the fault, based on characteristics observed in a stacked section. His line drawing of the major events in that section is given in Figure 2. Long observed differences in the densities of

Presented at the 54th Annual International Meeting, Society of Exploration Geophysicists, Atlanta. Manuscript received by the Editor June 2, 1987; revised manuscript received July $27,1987$.

*Formerly Seismological Laboratory, California Institute of Technology, Pasadena, CA 91125 ; presently Department of Geosciences, The Pennsylvania State University, University Park, PA 16802.

†tudes-Production Schlumberger, Clamant, France.

(1988 Society of Exploration Geophysicists. All rights reserved. 
events in different parts of the stacked section, relating changes in event density to crustal discontinuities. He interpreted diffractions at shallow levels of the fault zone as the effect of structures truncated by processes of brittle fracture. The deeper, "transparent" part of the zone represents a region of ductile flow. Because of the poor quality of the stack, these conclusions could not be made with confidence.

A better approach was undertaken by McBride and Brown (1986). They presented a complete reworking of the data set, facilitated by the previously unavailable, detailed control of the data processing and reduction. Precorrelation and prestack balancing, filtering, and editing contribute to an overall improvement in the stacked section. The densities of stacked events were again used to make associations with regionally known crustal structures.

This kind of interpretation is limited in that it is based on the assumptions in the stacking process, which, as Feng and McEvilly (1983) showed, are thoroughly violated in this region. Further, it attempts to assign geologic interpretations to a physical phenomenon, i.e., stacked event density, where little experimental control exists on the relation of particular geologic units to observable reflections. It is difficult to show that the stacked event density is not an artifact of the survey procedure or the data analysis, especially in an area where stacking could be invalid.

In fact, the methods used by Long (1981) and McBride and Brown (1986) are not capable of imaging the strongest reflection events in the data set. While these events cannot be analyzed by stacking, they can be reduced through a simple, though time-consuming, procedure. A multioffset Kirchhoff summation imaging process does succeed in showing where the major physical boundaries of the fault zone lie.

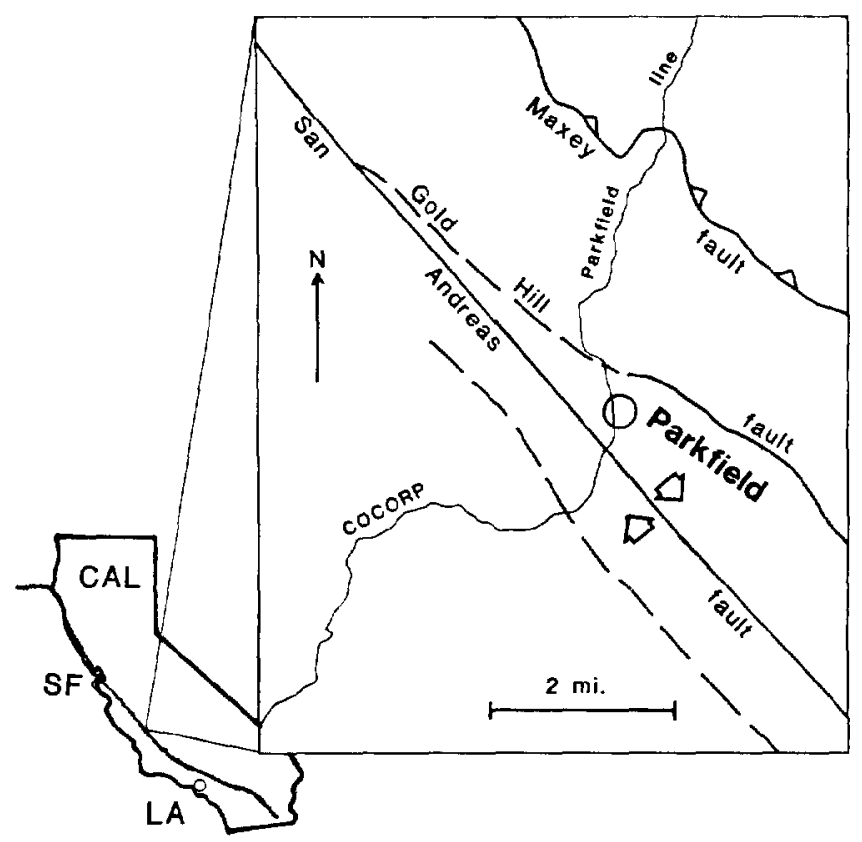

FIG. 1. Map of the vicinity of the town of Parkfield, showing the route of the COCORP survey and fault traces taken from Hanna et al. (1972).

\section{CHARACTERISTICS OF THE SURVEY}

The 1977 COCORP survey employed a 48 trace, $4.7 \mathrm{~km}$ long off-end receiver spread with a minimum offset of $0.45 \mathrm{~km}$. The vibrators started at the southwest end of the line and pushed the spread to the northeast, with shots at $100 \mathrm{~m}$ intervals. The line is, however, sinuous over distances smaller than the length of the receiver spread, as shown by Figure 1. This sinuosity has the effect of spreading the midpoints of the recordings from different pairs of sources and receivers over a substantial area, as discussed by McBride and Brown (1986). In particular, the midpoints of receivers at shorter offsets are close to the line, while the long-offset midpoints are most distant, generally following a straighter path than the line. This kind of geometric discrepancy is damaging to the stacking process, since short-offset raypaths may pass through different near-surface heterogeneities than the long-offset raypaths. The situation offers some advantages, however, for true three-dimensional (3-D) analysis techniques, since the line's sinuosity provides some 3 -D coverage.

A large number of field records distributed along the entire line were examined. By far the strongest, most coherent events observable in the multioffset data appeared near the San Andreas fault. These events show horizontal or reverse moveouts, in that their arrival times decrease with increasing offset and have amplitudes comparable to the direct arrivals (Figure 3). The events are found on all of the reasonably clean gathers near the fault. The timing and apparent velocities of the events change rapidly as the orientation of the survey line changes. These factors suggest that the events may have originated as sidewall reflections from steeply dipping structures. Similar events were observed by Robinson (1945) in reflection surveys on the Gulf Coast. He interpreted them as horizontally propagating refractions reflected from lateral discontinuities in the refracting structures, probably faults. From a number of sur-

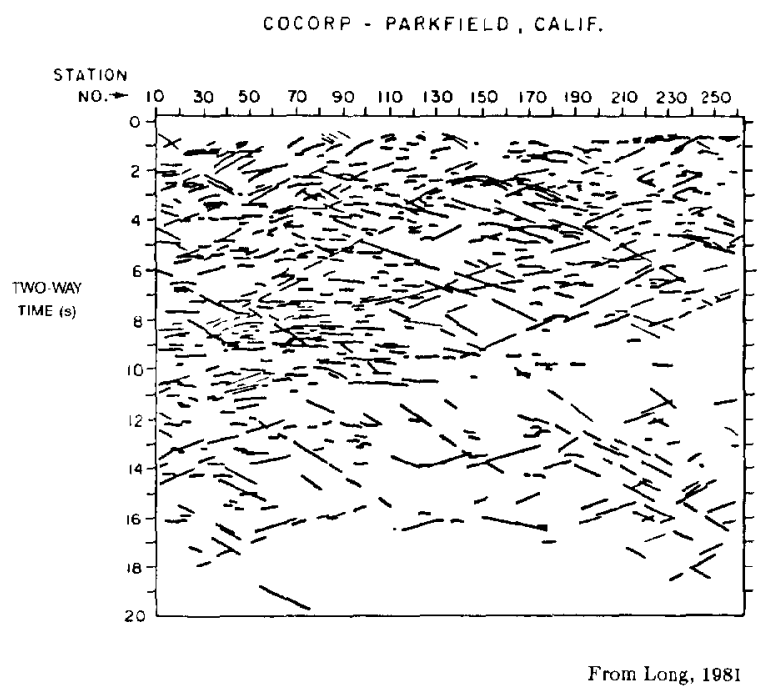

Fig. 2. Line drawing by Long (1981) of a COCORP preliminary stack of the Parkfield survey. The station numbers increase from southwest to northeast, with the San Andreas fault near station 140 . 
veys with different orientations, he was able to locate the faults by relating the arrival times of the events to the propagation time along the refractor.

Some compensation for the sinuosity of the COCORP line can be made by sorting out common-midpoint (CMP) gathers of the data. All traces whose midpoint fell within $230 \mathrm{~m}$ of a node of a two-dimensional (2-D) grid of points with the same spacing were sorted into a gather for that node, regardless of the orientation of the shot-receiver pair. Two of these gathers are shown in Figure 4. The unusual arrivals at about $2 \mathrm{~s}$ at the farther offsets can be found on many gathers. As Figure 4 shows, the apparent velocity of the sidewall reflection changes drastically as the survey crosses the San Andreas. On the southwest side, the receivers are between the vibrators and the fault, so the moveout is negative. On the northeast side, the receivers are farther from the fault than the vibrators, so the moveout is normal (positive).

Such events, especially where they have negative moveout, obviously cannot be stacked using any physically meaningful stacking velocity. The stacking process would destroy their coherency, rendering them invisible in a stacked section. On the other hand, where the line is oriented such that the sidewall reflection has a normal, positive moveout, it may stack coherently, but its location in the section will be completely incorrect. If the reflection point is not in the plane of the survey line, it will not be possible to migrate the stacked reflection to its correct location. Yet some process of imaging the reflector producing these events must be found, since they carry most of the energy in the seisimic gathers. They therefore represent the most fundamental physical boundaries in the area.

To obtain an image of these reflectors, it is necessary to have a starting idea of the velocity structure in the area. The CMP gathers made from the southwest part of the line did show coherent reflections from near-horizontal structures in the upper $3 \mathrm{~km}$ of the crust. Interval velocities calculated from velocity semblances of these gathers indicate a strong velocity gradient in this area similar to that found by Liu (1983) from seismicity analysis. The gradient, shown in Figure 5, also incorporates deeper velocity information derived from refraction surveys by Eaton et al. (1970). These profiles suggest that the strong lateral heterogeneity across the San Andreas is limited to velocities in the upper $5 \mathrm{~km}$ of the crust.

The velocity gradient in the uppermost crust explains how reflections from a vertically oriented structure could be recorded by a horizontally oriented receiver spread. Horizontal bending of the raypaths with depth, within such a strong velocity gradient, assures that reflections can be located on structures dipping even more than 90 degrees from the horizontal. The ray bending will, unfortunately, also act to limit the range of depths covered by the recorded reflections.

\section{IMAGING METHOD}

Given the constant-to-reverse moveout exhibited by the arrivals on the CMP gathers, it is clear that stacking the traces

\section{COCORP CORRPIIATIPD DATA}

\section{Northeast}

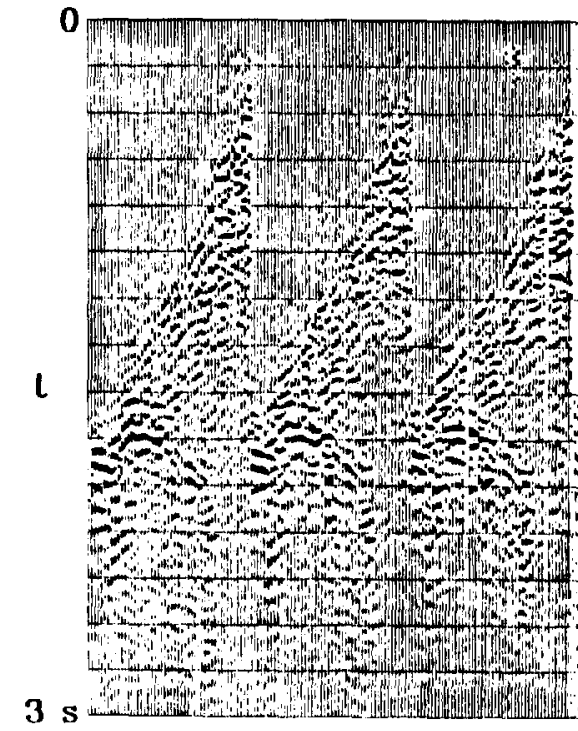

\section{San Andreas Fault}

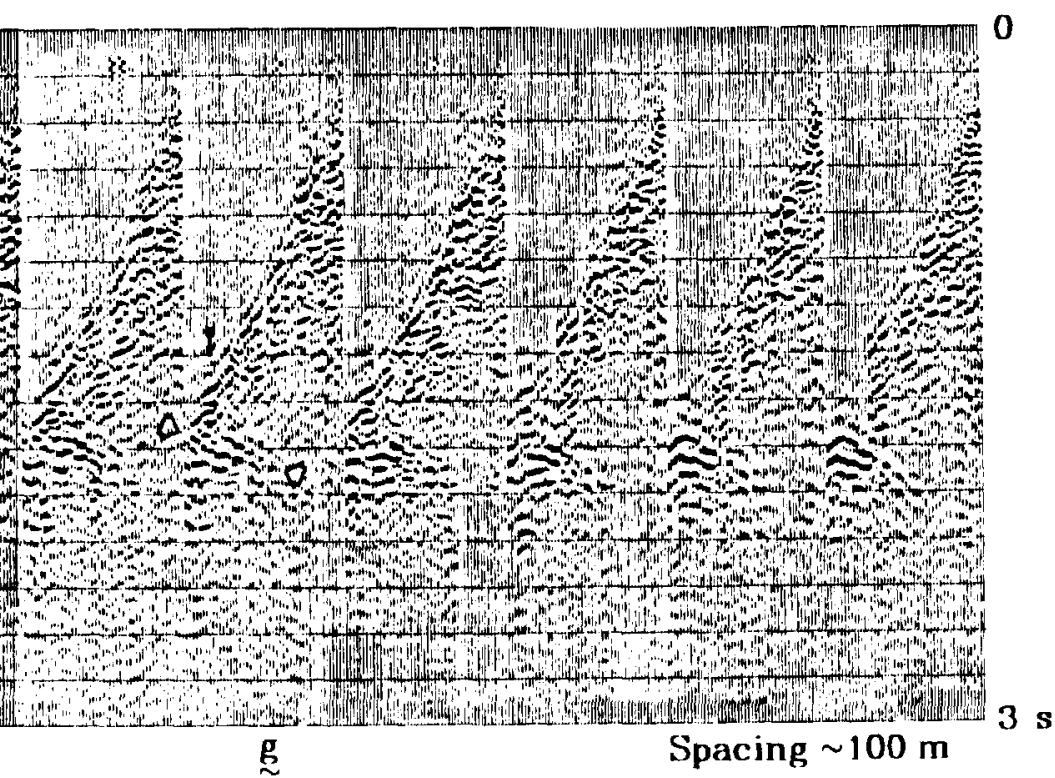

FIG. 3. Consecutive raw common-shot gathers recorded by COCORP in 1977. No amplitude balancing has been applied to these correlated vibrator recordings. Each of the nine panels contains 48 traces from a $4.7 \mathrm{~km}$ off-end receiver spread being pushed by the vibrators toward the northeast with a minimum offset of $0.45 \mathrm{~km}$. The sources are $0.1 \mathrm{~km}$ apart. In the center panel, the receiver closest to the San Andreas fault trace is indicated by the vertical arrow. The open arrows indicate the strongest arrival on the records; its arrival time decreases with increasing offset. 
could not help image the structure that produced the arrival. Such a situation demands prestack migration. The method must be simple enough to enable tens of thousands of traces to be processed in a reasonable amount of time. Yet it should be robust enough to prevent being adversely affected by the copious random and coherent noise seen on such deep crustal surveys

\section{Approximations to the wave equation}

Some assumptions about the data greatly simplify the task of inverting an elastic wave field for the properties of the earth through which it has propagated. Le Bras (1985) developed representations of the acoustic and elastic waye equations, based on several assumptions which reduced the inversion of reflection data to a process very similar to the Kirchhoff sum migration of Jain and Wren (1980). First, the Born approximation considers the scattered wave field to result from small, rapid variations in material properties, which are superimposed on larger, slowly varying properties which affect only the propagation of the wave. This approximation allows the effect of scattering at varying incidence angles to be linearized (Wu and Aki, 1985). Second, the WKBJ approximation, which assumes that the medium parameters vary slowly along the

\section{COCORP CORRELATED DATA}

\section{Common-Midpoint Gathers True Amplitude}
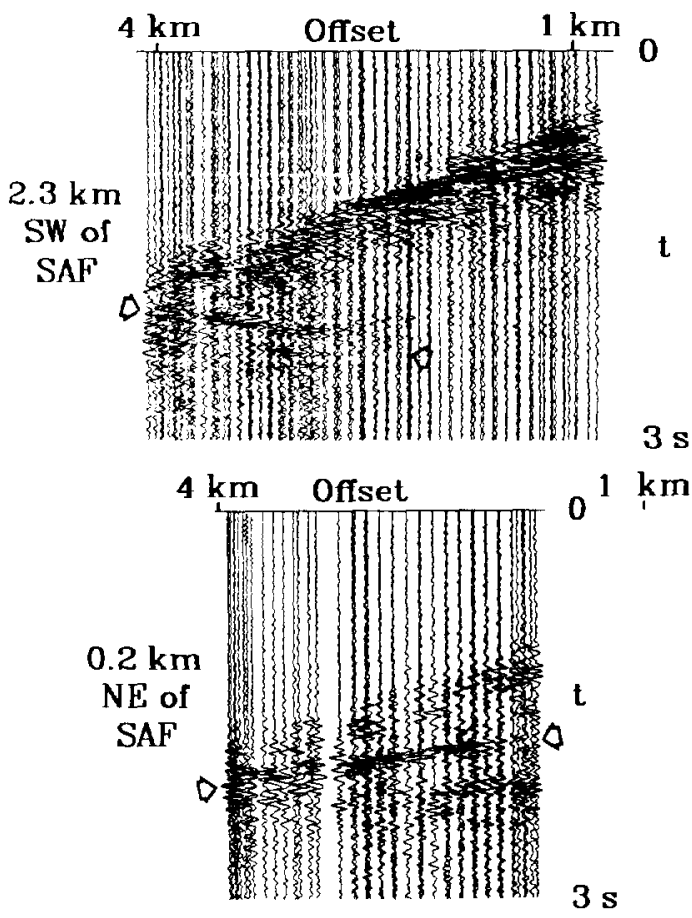

FIG. 4. Two CMP gathers of unequalized traces assembled within 2-D bins $460 \mathrm{~m}$ wide at different locations near the $S a n$ Andreas. The open arrows indicate the positions of a strong reflection from the steeply dipping fault zone. The timing and apparent velocity of the sidewall reflection change as the geometric relationship of the shots and receivers relative to the fault changes. propagation path, allows the propagation through the medium to be regarded as a high-frequency ray. This approximation is also dependent upon the third approximation, that the source and receiver are in the far field relative to the reflector.

With these three approximations the data can be considered to be a linear superposition of rays from individual point scatterers. The tomographic approximation to the inverse of this superposition, as discussed by Le Bras (1985), is simply the superposition of rays from individually recorded reflections. Thus, the scattering potential of the medium can be estimated as the sum of the reflections recorded by each source-receiver pair, positioned according to the traveltime of the rays between the surface points and the subsurface reflector.

Since the purpose of this paper is simply to establish the geometry of the scatterers within the medium, we ignore the amplitude correction factors due to the angle of incidence on the scatterer and to the length of the travel path. Further, the scatterer is represented by the sum of reflection wavelets without crosscorrelation with the source wavelet, since a source wavelet is not available. Certain restrictions apply; the data being inverted should contain only primary $P$-to- $P$ wave reflections without any postcritical angle reflections or refractions.

With these approximations, the Kirchhoff summation method used here to image the geometric distribution of acoustic reflectivity is very similar to that used by McMechan and Fuis (1987) and outlined by Jain and Wren (1980). Figure 6 shows the geometry of a 3-D reflection from a steeply dipping fault zone within a vertical velocity gradient. This method is especially versatile in that the reflectivity at any depth point may be inverted from data recorded from sources and receivers at any location. The ordering of the data and of the inversion points are immaterial, since the tomographic sum may be made in any order.

Figure 7 summarizes the imaging procedure. Unsorted seis-

\section{VELOCITY PROFILES}

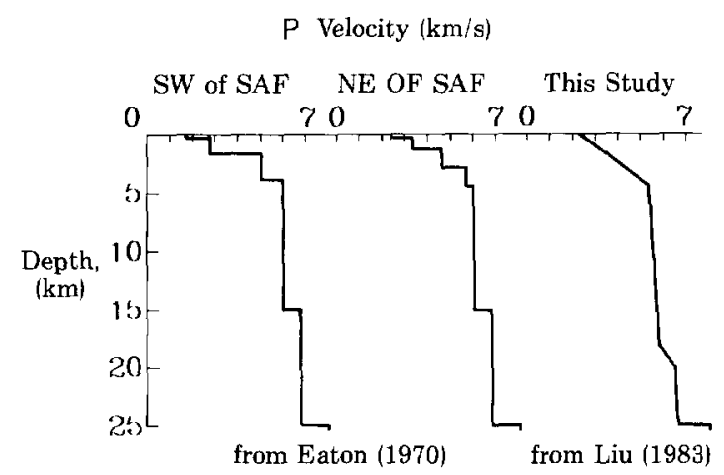

FiG. 5. Crustal velocity profiles derived for the blocks on each side of the San Andreas from refraction surveys (Eaton et al., $1970)$ and as modified by Liu (1983) with arrival times from the local seismicity. The gradient at the top of Liu's profile was adjusted to agree with velocities derived from the moveout of reflections recorded by the COCORP survey to the southwest of the San Andreas. 


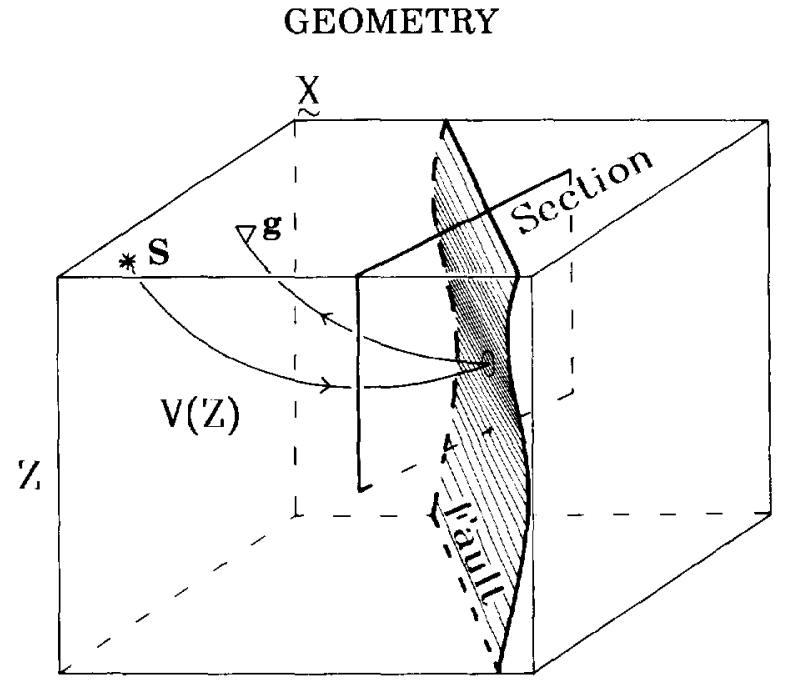

FIG. 6. Geometry of a sidewall reflection from a fault zone within a medium showing an increase in velocity with depth $Z$. S and $g$ are the source and receiver positions, respectively. mogram traces are mapped into a depth section by computing the traveltime from the source to the depth point and back to the receiver. A velocity model that varies only with depth is used, as derived from the velocity semblances and the model of Liu (1983; Figure 5). The traveltime calculation included turning rays, which allows the imaging of structures with dips greater than 90 degrees. To allow for propagation through a laterally heterogeneous medium, the traveltime calculation could take the form of ray tracing through a variable-velocity medium. If, however, the bulk of the travel path can be constrained to a part of the medium in which velocity varies principally with depth, then a simple ray tracing through a vertically varying medium can be used for data with any orientation of the source-receiver offset. This allows the traveltimes to be calculated only once for the range of offset and depth of the experiment, greatly speeding the imaging process. For this reason the migrations performed here employ mainly sources and receivers on just one side of a major lateral discontinuity such as the San Andreas.

Once the traveltime down to and up from the depth point has been obtained, the value of the seismogram at that time is summed into the section at the depth point. Spurious ares due to noise bursts and badly gained traces are easily identified from plots of the shape of the wavefront for the given velocity model. Since small sections in areas of particular interest can be migrated one at a time, and since storage of large numbers

\title{
KIRCHHOI'P'-SUM ALGORITHM
}

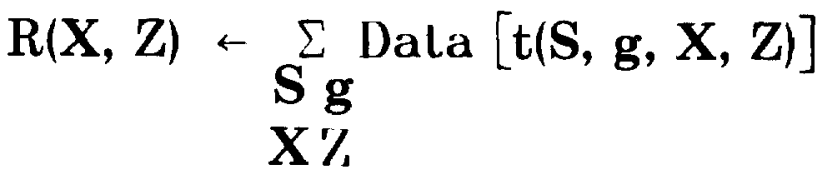

\section{Data Volume}

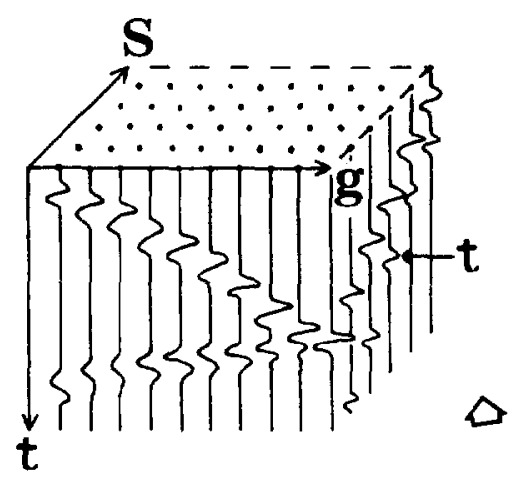

Migrated Section

\section{livaluale 'l'raveltime

$$
\mathrm{t}=\mathrm{t}(\mathbf{S}, \mathbf{g}, \mathbf{X}, \mathbf{Z})
$$

FIG. 7. Schematic representation of a Kirchhoff sum algorithm used to form a migrated image of multioffset reflection data. For each recorded source-receiver pair $(S, g)$ over each point $(X, Z)$ in some depth section, a traveltime is calculated. The amplitude of the recorded trace at that time is then summed into the point of the depth section. 


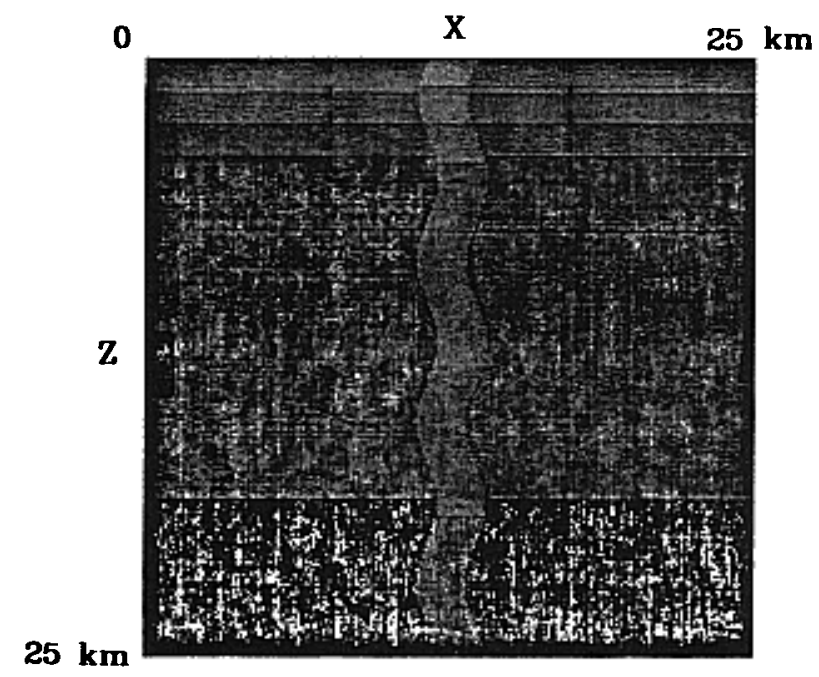

FIG. 8. 2-D velocity model representing a cross-section $25 \mathrm{~km}$ wide by $25 \mathrm{~km}$ deep across a fault zone having a sinuous profile. The $2 \mathrm{~km}$ wide zone has a velocity 20 percent lower than in the adjacent regions. Outside the fault zone, the model is that of Liu (1983, Figure 5), which varies only with depth. The dashed box is the region imaged in Figures 11 and 12. of traces is not necessary, this method is economical even on a relatively small computer. If the seismic survey has sufficient 3-D coverage, the reflectors can be easily imaged in three dimensions by properly locating the depth sections.

To test the method, a survey was simulated over an idealized model of a steeply dipping fault zone. A finite-difference solution of the 2-D acoustic wave equation was used. This solution included all acoustic multiples, postcritical reflections, and refractions. The velocity model was identical to the one identified in Figure 5, except that a $2 \mathrm{~km}$ wide fault zone having a 20 percent lower velocity at a given depth, and a sinuous profile in cross-section, was introduced to test the ability of the method to resolve vertically complex fault geometries (Figure 8). Synthetics were calculated with shot and receiver spacings meant to simulate the Parkfield survey, but with poorer coverage. The synthetic gathers, given in Figure 9, show strong reflections from both sides of the near-vertical fault zone, which are quite similar to the arrivals in the Parkfield data. The traveltimes calculated for the velocity model to points at different depths and offsets, which were also used for the inversions, are given in Figure 10.

The effect of the Kirchhoff sum migration can be illustrated by migrating a single trace. This image is shown by Figure 11. Each part of the trace, after muting of the first arrival, has been back projected into the depth section along contours of equal traveltime similar to those in Figure 10. At least one point of this projected image is correctly located. As the back projections of more traces are summed into the image, the correctly located point should be reinforced, while the incorrectly located parts of the arcs should be canceled by destructive interference.

Summing the back projections of all 15 of the 48-trace syn-

\section{SYNTIIETIC GATIIL'RS}

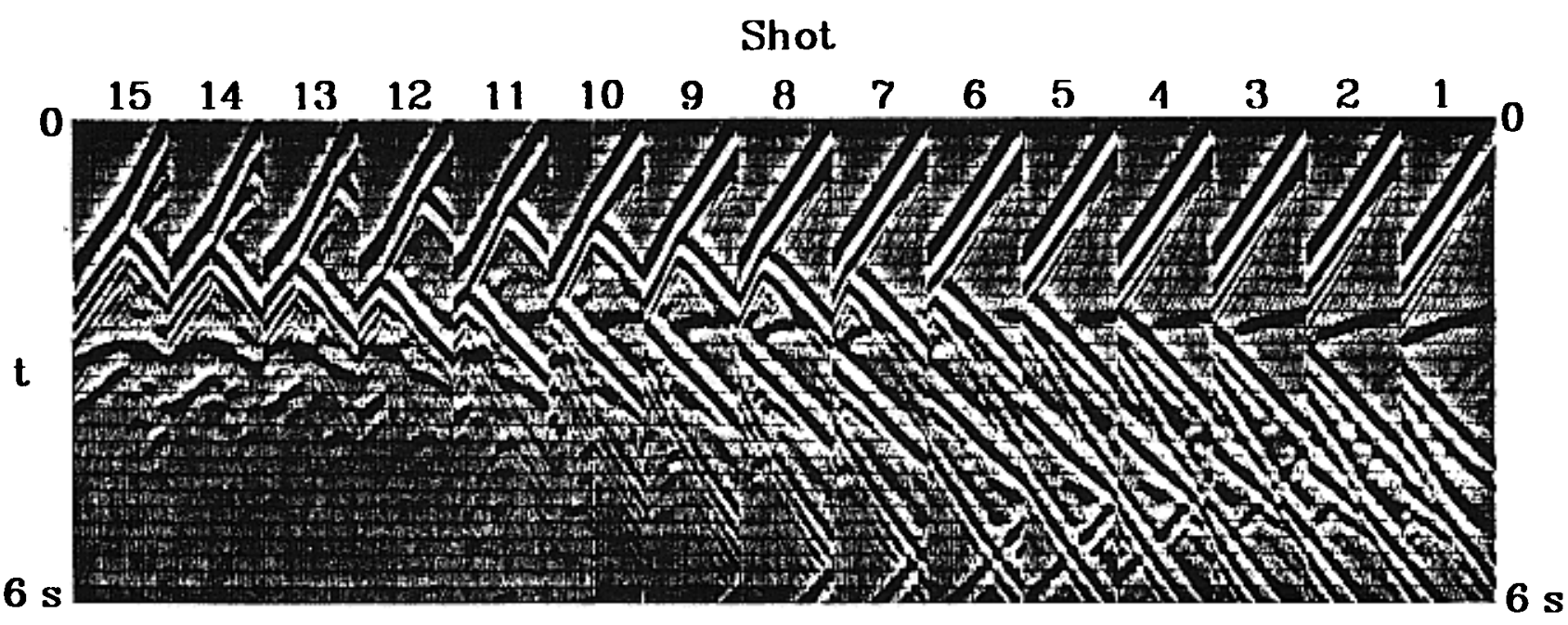

$\mathbf{g}$

FIG. 9. 2-D acoustic finite-difference synthetics generated from the velocity model of Figure 8 . The geometry of the synthetic spread is similar to the geometry of the COCORP survey. The common-shot gathers are presented as they were in Figure 3. Note the reflection showing normal hyperbolic moveout at $2.5 \mathrm{~s}$ from shots 1 through 5 , and the strong sidewall reflections from the fault zone showing negative moveout and intersecting the first arrival. 


\section{'TRAVELTIME' MA'TRIX}

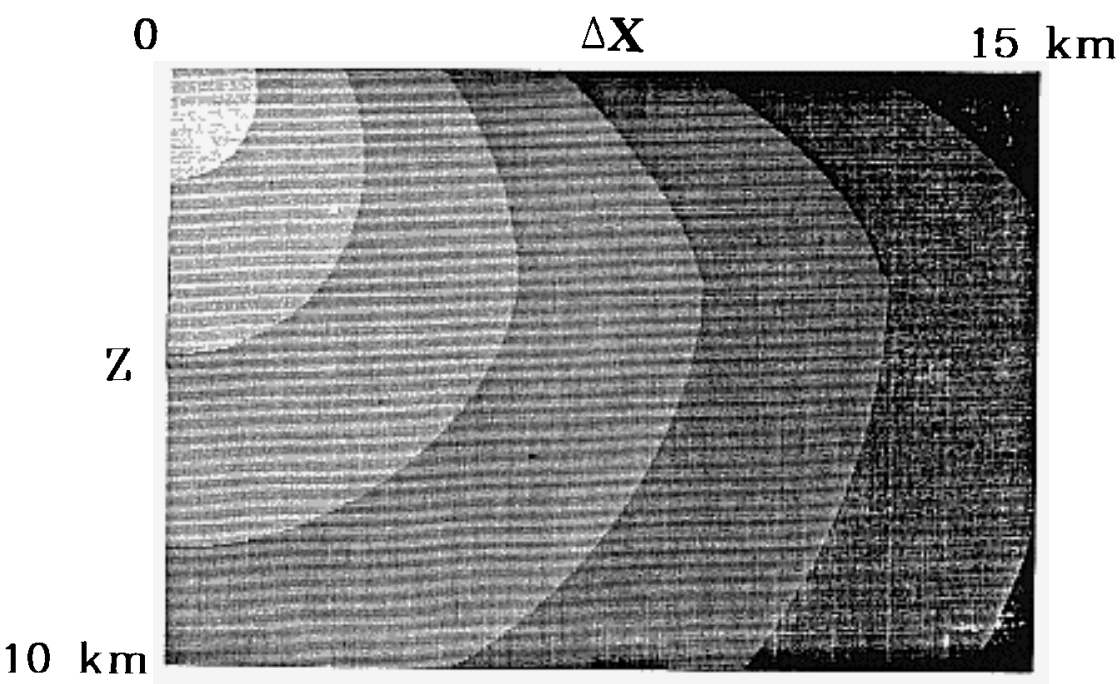

FIG. 10. Matrix of traveltimes from a source in the upper left corner to any point in a cross-section of depth $Z$ against offset $\Delta \mathbf{X}$, calculated by ray tracing through the vertically varying velocity model given in Liu (Figure 5). Darker areas have the longer traveltimes, with the contours on equal traveltime.

\section{MIGRATION OF SYNTHE"IIC Single Trace}

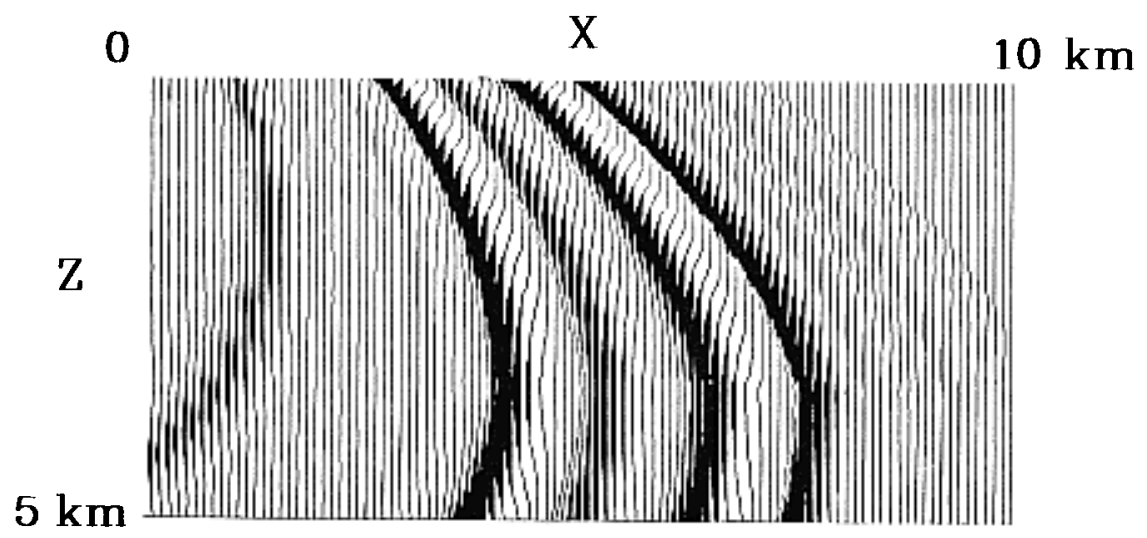

FiG. 11. Depth section located in dashed box of Figure 8 with the Kirchhoff sum migration of a single trace. Note the similarity to the shape of traveltime contours of Figure 10. 


\section{MIGRATION OF SYNTHETICS}

\section{Gathers}

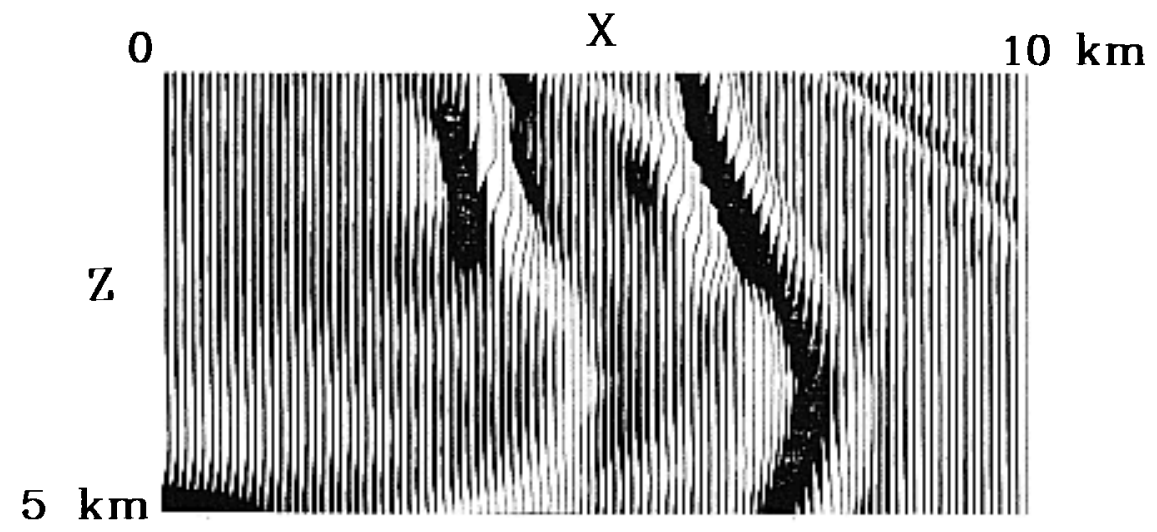

Fig. 12. Full Kirchhoff migration of 720 traces of 15 synthetic shot gathers within the dashed box of Figure 8 . Note the resolution of the sinuous shape of the fault zone, including portions imaged by rays bent more than 90 degrees from vertical.

thetic gathers produced the image in Figure 12. This image should be a reconstruction of the part of the velocity model in Figure 8 set off by the dashed line. The sinuous geometry of the zone has been reconstructed quite well. In the lower one-

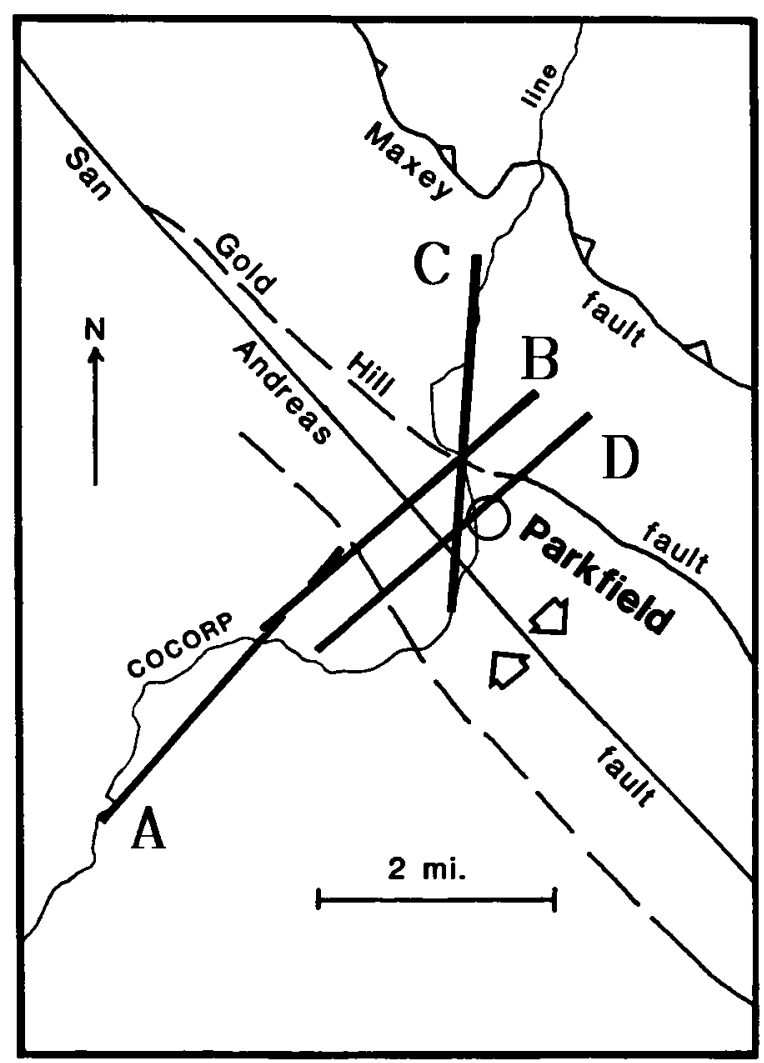

FIG. 13. Map of the vicinity of Parkfield showing the locations of the four depth sections migrated from the COCORP shot records. Each section is $5 \mathrm{~km}$ long by $5 \mathrm{~km}$ deep. third of the image, however, the reconstruction is not complete due to the lack of reflection points on the fault zone at those depths. Because of the strong velocity gradient in the first few kilometers of depth, most of the rays turn horizontally or refract at shallow depths. In fact, some of the most strongly reconstructed points lie along refractors that prevail at particular depths.

\section{MIGRATION RESULTS}

Despite all the approximations, the poor quality of the records, and the large uncertainties in the velocity model, images can be obtained from the actual COCORP survey data. The reflections to be back projected are clear, highamplitude events that are most prominent on the records in Figures 3 and 4. Because of the high signal-to-noise ratio, there can be fair confidence in this case that the migrated images will not be dominated by the effects of spurious, highamplitude noise.

The reflection data are back projected into four depth sections whose locations are shown on Figure 13. The sections were located where there are heavier concentrations of midpoints, with $B$ and $D$ made parallel to test 3-D aspects of the image. All back projections were made using traveltimes calculated from the velocity profile in Figure 5, which is most appropriate for the region to the southwest of the San Andreas. In migrating all of the sections (except for $\mathrm{C}$ ), only traces having both sources and receivers southwest of the Gold Hill fault were used. Section B was migrated both from traces having the first arrivals muted and from traces without any mutes. The two sections showed little difference, so all of the migrations were run on unmuted data. However, ignoring the nearest traces, as was done by McBride and Brown (1986), did improve the imaging. 
The back-projected depth sections are shown in Figure 14. Many parts of these images are artifacts. Where the geometric coverage is poor, because of the layout of the line or the concentration of raypaths along refracting structures, the arching tails of the individual back-projected events may not be canceled. It is most useful to look for strong images that are at least crossed by similar ares. Among the images in which we have some confidence is, in section $\mathrm{D}$, a reflector dipping at least 45 degrees to the southwest underneath the San Andreas fault, possibly extending to the surface near the trace of the Gold Hill fault. It can be found to a depth of at least $4 \mathrm{~km}$. Section $B$ contains a similar reflector, although it is less well-defined. This reflector also appears on section $\mathrm{C}$, with a shallower apparent dip. A subhorizontal reflector is observed at a depth of about $3 \mathrm{~km}$ between the surface traces of the San Andreas and an unnamed fault to the southwest on section B. Such a reflector is also present in section D. A strong near-vertical reflector is shown in section $\mathrm{B}$, extending from the surface to a depth of about $1 \mathrm{~km}$. This reflector can also be discerned in section $A$, where it appears to mark the truncation of a strong subhorizontal reflector at a depth of 2 $\mathrm{km}$. which continues to the southwest.

Hanna et al. (1972) have synthesized the surface geology of the Parkfield area with gravity and magnetic data. Figure 15 interprets the location of the imaged reflectors and their association with mapped surface features and suspected geologic relations. In making interpretations of the connection of reflectors to geologic boundaries, the most obvious, simple boundaries that would provide the greatest velocity contrast should be stressed. The Gold Hill fault incorporates slices of serpentinized ultramafic rocks from the metamorphic Franciscan complex to the northeast and juxtaposes them against crystalline rocks of intermediate to mafic composition which crop out to the southwest. Such a contrast would create a strong reflector. Sections $B$ and D indicate that the fault may well dip steeply to the southwest and intersect the vertically dipping San Andreas at a depth of 3 to $4 \mathrm{~km}$. Between the San Andreas and the unnamed fault to the southwest, the intermediate-ultramafic contact may be subhorizontal at a depth of $3 \mathrm{~km}$. It appears to be truncated by the southwestern fault against the possibly granitic basement of the Salinian block. The same fault may also truncate a $2 \mathrm{~km}$ thick Tertiary sedimentary section to the southwest against shallow crystalline rocks of intermediate composition in the fault zone, thus producing the strong vertical reflector. A strong reflection is apparently not observed from the active trace of the San Andreas itself, indicating that this trace may cut through relatively uniform mafic to intermediate rocks caught in the fault zone above $3 \mathrm{~km}$.

The use of data from the southwest side of the San Andreas, combined with the presence of granitic and sedimentary rocks in the fault zone having affinities to the block to the south-

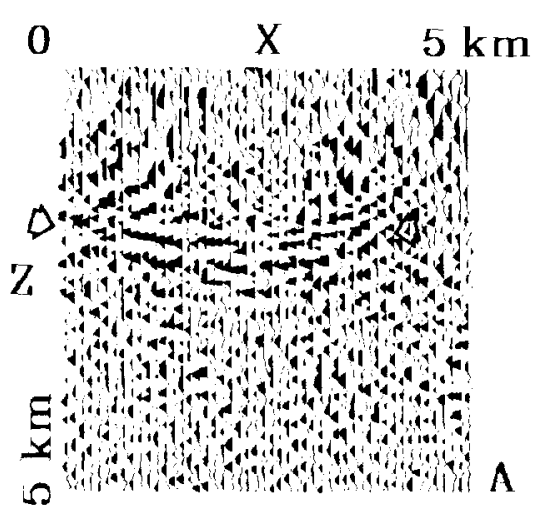

Southwest

\section{MIGRATIONS}

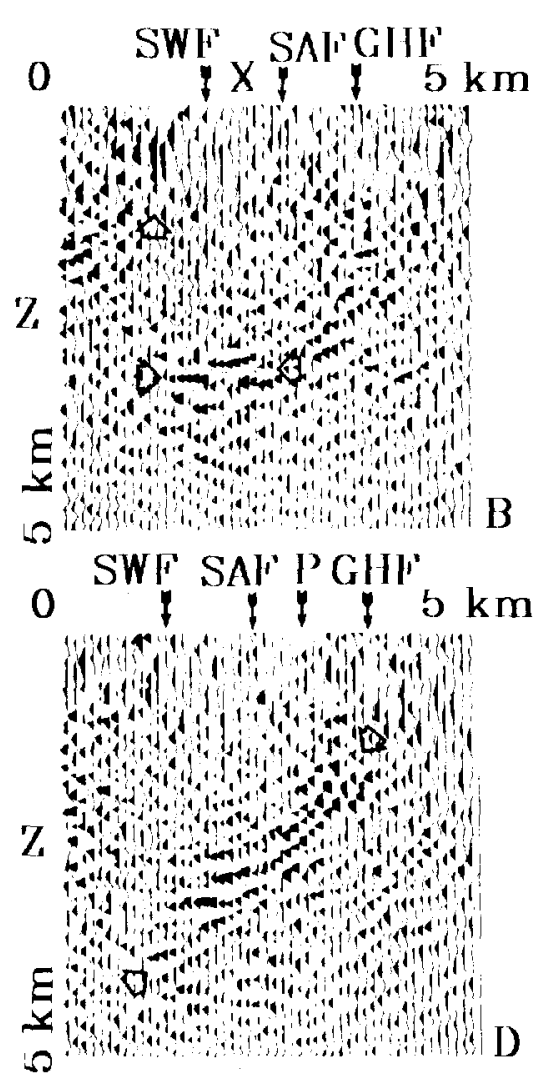

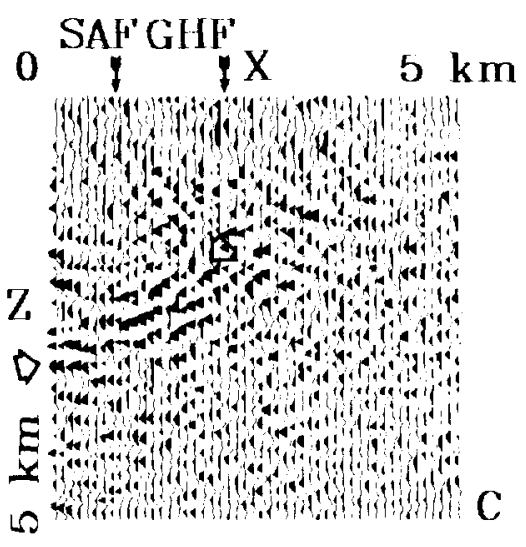

Northeast

Fig. 14. Kirchhoff sum migrations of the COCORP shot records in the depth sections shown in Figure 13. The positions on the surface of the unnamed fault to the southwest of the San Andreas (SWF), the San Andreas fault (SAF), and the Gold Hill fault (GHF) are indicated by arrows. Other arrows indicate the reflectors interpreted in the text. 


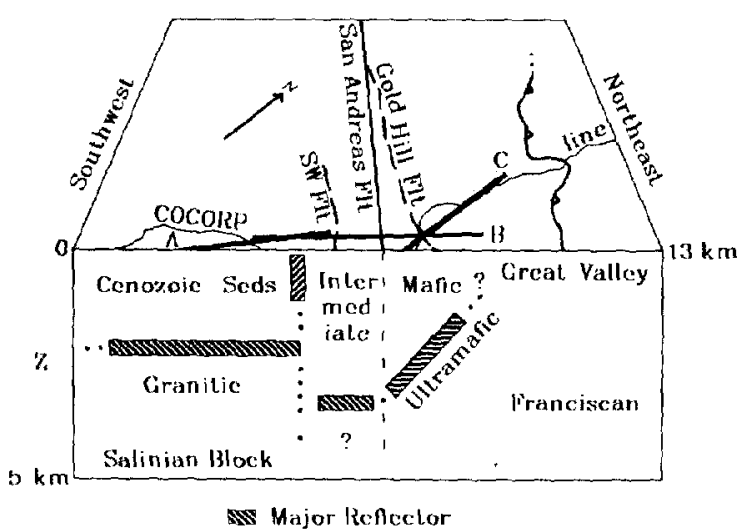

Fig. 15. Perspective block diagram showing mapped features of the Parkfield region in relation to a sectional view of the positions of the major reflectors imaged by the Kirchhoff sum migrations of the COCORP shot records.

west, allowed the laterally homogeneous velocity model to produce fairly accurate reconstructions, at least above the ultramafic rocks. Most of the major reflectors have probably been located to within $\pm 0.5 \mathrm{~km}$. Any imaging of deeper reflectors will require the use of data far enough away from the fault zone to avoid the complications in the upper several kilometers.

\section{CONCLUSIONS}

It has been shown that a 3-D Kirchhof sum inigration before stack can image steeply dipping reflectors which produced arrivals that cannot be stacked with conventional CMP methods. Such reflections can be observed in the data from a 1977 COCORP survey across the San Andreas fault near Parkfield, California. Previous workers, who used conventional methods, could not interpret the events, which arose from strong lateral heterogeneities. However, applying the Born, WKBJ, and far-field approximations to the wave equation simplifies the inversion of these reflections to a ray-equation back-projection process very similar to Kirchhoff sum migration. This process can be easily implemented on unsorted data to back project reflector images into arbitrarily oriented depth sections of limited size. The process was verified by inversions of full-wave acoustic synthetics incorporating the same limitations of geometric coverage inherent in the COCORP survey. Although limited in accuracy by the poor geometric coverage and the laterally inhomogeneous velocity structure, the method imaged reflectors around the San Andreas fault that are consistent with the known structural features of the area. The imaged relationships suggest that the fault zone bas juxtaposed mafic to intermediate crystalline rocks against Cenozoic sediments on the southwest and serpentinized ultramafics on the northeast. The modern trace of the San Andreas fault does not form a reflector strong enough to be imaged. Such information can prove useful in efforts to reconstruct the complex history of the motion of crustal blocks caught in the transform zone.

Future work will be aimed at testing the method on additional structures as data become available, and at taking advantage of the amplitude information available in the multioffset domain to constrain the inversion even further.

\section{ACKNOWLEDGMENTS}

Digital data from the COCORP Parkfield survey were kindly provided by $\mathrm{S}$. Kaufman of Cornell University. The work was supported by a generous fellowship from the Amoco Foundation and by grants from Sun Oil Co. and the Keck Foundation.

Contribution no. 4475, Division of Geological and Planetary Sciences, California Institute of Technology.

\section{REFERENCES}

Eaton. J. P., D'Neill, M. E., and Murdock, J. N., 1970, Aftershocks of the 1966 Parkfield-Cholame, California, earthquake: a detailed study: Bull., Seis. Soc. Am., 60, 115 -1197.

Feng, R., and McEvilly, T, V., 1983, Interpretation of seismic refleciion proffing data for the structure of the San Andreas fault zone: Bull,, Seis. Soc. Am., 73, 1701-1720.

Hanna, W. F., Burch, S. H., and Dibblee, T. W., 1972, Gravity, magnetics, and geology of the San Andreas fault area near Cholame. California: U. S. Geol. Surv. Prof. Paper 646-C

Jain, S.. and Wren, A. E., 1980, Migration before stack-Procedure and significance: Geophysics, 45, 204-212.

Le Bras. R., 1985. Methods of multiparameter inversion of seismic data using the acoustic and elastic Born approximations: Ph.D. thesis, Calif. Inst. of Tech.

Liu, H-L., 1983, Interpretation of near-source ground motion and implications: Ph.D. thesis, Calif. Inst. of Tech.

Long. G. H., 1981, A COCORP deep seismic reflection profile across the Sitn Andreas fault, Parkfield, California: M.Sc. thesis, Comell Univ.

MeBride, J. H., and Brown, L. D., 1986, Reanalysis of the COCORP deep seismic reflection profile across the San Andreds fault, Parkfield, California: Bull., Seis. Soc. Am., 76, 16681686.

MeMechan, G. A., and Fuis, G. S., 1987, Ray equation migration of wide-angle reflections from southern Alaska: J. Geophys. Res., 92 $407-420$.

Robinson, W. B., 1945, Refraction waves reflected from a fault zone: Geophysies, 10, 535. 545

Wu, R., and Aki, K. 1985, Scattering characteristics of elastic waves by an elastie heterogeneity: Geophysics, $50,582-595$. 